\title{
BMJ Open Excess mortality in adults with documented diabetes in Germany: routine data analysis of all insurance claims in Germany 2013-2014
}

Christian Schmidt (D) , Lukas Reitzle, Christin Heidemann (D), Rebecca Paprott (D), Thomas Ziese, Christa Scheidt-Nave, Jens Baumert (1)

To cite: Schmidt C, Reitzle L, Heidemann C, et al. Excess mortality in adults with documented diabetes in Germany: routine data analysis of all insurance claims in Germany 2013-2014. BMJ Open 2021;11:e041508. doi:10.1136/ bmjopen-2020-041508

- Prepublication history for this paper is available online. To view these files, please visit the journal online (http://dx.doi. org/10.1136/bmjopen-2020041508).

Received 10 June 2020 Revised 16 December 2020 Accepted 17 December 2020

D) Check for updates

(c) Author(s) (or their employer(s)) 2021. Re-use permitted under CC BY-NC. No commercial re-use. See rights and permissions. Published by BMJ.

Department of Epidemiology and Health Monitoring, Robert Koch Institute, Berlin, Germany

Correspondence to Dr Christian Schmidt; schmidtchri@rki.de

\section{ABSTRACT}

Objectives Little is known about the age-specific excess mortality pattern of people with diagnosed diabetes in Germany. Thus, our goal was to determine the excess mortality in diagnosed diabetes overall and stratified by age and sex based on claims data.

Design Routine data analysis using a claims dataset from all statutory health-insured persons in Germany in 2013, which accounts for about $90 \%$ of the population.

Participants We included persons who lived in Germany, were insured at least 360 days, were not self-paying any health services and were aged 30 years or older leading to a total number of 47.3 million insured persons for analyses.

Exposure Diabetes was determined by the International Classification of Diseases-10 codes E10-E14, which were documented in 2013 in at least two-quarters on an outpatient setting or at least once on an inpatient setting. Outcome measures The vital status in the study population was drawn from the claims dataset for the year 2014. We derived the excess mortality estimated as an age-adjusted mortality rate ratio (MRR) by sex and for age groups using a Poisson model.

Main results We found age-adjusted MRRs $(95 \% \mathrm{Cl})$ for diabetes of 1.52 (1.51 to 1.52) for women and 1.56 (1.56 to 1.56) for men. These figures declined with increasing age and were highest for ages 30-34 years with 6.76 (4.99 to 9.15) for women and 6.87 (5.46 to 8.64) for men, and lowest for age 95 years and older with 1.13 (1.10 to 1.15) for women and 1.11 (1.05 to 1.17) for men. Conclusions We derived deeply age-stratified figures on excess mortality in diabetes for Germany. Establishing a sustainable analysis of excess mortality is aimed at within the framework of diabetes surveillance.

\section{INTRODUCTION}

Diabetes mellitus is a chronic metabolic disease of high public health impact in Germany and worldwide. ${ }^{1}$ According to the Global Burden of Disease Study 2017, diabetes ranks among the top 10 leading causes of death globally. ${ }^{2}$ Available treatment with insulin and glucoselowering drugs has greatly reduced the risk of acute complications and premature mortality.
Strengths and limitations of this study

- This is the first study in Germany, which analyses excess mortality of diabetes on the basis of routine data covering almost the entire German population.

- The completeness of the study data in terms of deaths and documented diagnosis allows calculating nearly unbiased and deep-stratified diabetesrelated mortality.

- We have not distinguished the type of diabetes because routine data contain implausible double diagnoses of type 1 and type 2 diabetes in the same person.

- The study data are limited to documented diagnoses, that is, no information about undiagnosed morbidity is available.

- In principle, the study data allow a continuous assessment of changes in mortality, which is suitable for public health surveillance.

Nevertheless, persons with diabetes still have a higher age-adjusted risk of death compared with persons without diabetes mainly because of an increased risk of microvascular and macrovascular complications. ${ }^{3}$

Monitoring diabetes-related mortality over time is an important part of national diabetes surveillance activities, as the agespecific excess risk of death among persons with diabetes compared with those without diabetes serves as an indicator of quality of diabetes care. Some countries, such as Sweden, Denmark and Scotland, have established National Diabetes Registers, and along with a legal basis for individual health data linkage, these data allow a reliable assessment of diabetes-related mortality in comparison with the general population ${ }^{45}$ or populationbased controls. ${ }^{6}$ Results from these countries consistently demonstrate a significantly higher risk of death in association with diabetes, but greatly vary with regard to the overall magnitude of excess risk as well as sex 
differences. A recent meta-analysis of diabetes-related allcause mortality based on 86 prospective cohorts showed a higher pooled adjusted relative risk of death among women than men (1.93 vs 1.74). ${ }^{7}$ The studies from Sweden as well as a further study from Australia have been age disaggregated, indicating that excess mortality among persons with type 2 diabetes significantly decreases with increasing chronological age. ${ }^{68}$

In Germany, a national diabetes surveillance system is currently being established at the Robert Koch Institute as the national public health institute. One of the main goals is to cover the diabetes-related mortality continuously ( www.diabsurv.rki.de). Over the past 20 years, a number of epidemiological studies in Germany have provided estimates of mortality rate ratios (MRRs) comparing mortality rates among persons with and without diabetes. The results from these studies vary due to differences in study design and study populations, methodological issues, regional versus national data, follow-up time, and insight from age-stratified and sex-stratified analyses is limited due to the small number of observations. ${ }^{9}$ In addition to these population-based estimates, a recent study estimated diabetes-related MRRs for the population 65-90 years of age in Germany based on mathematical modelling using official death statistics, and prevalence and incidence estimates derived from statutory health insurance (SHI) claims data. ${ }^{10}$ Due to partly conflicting findings stated above, further research is needed to increase knowledge on diabetes-related excess mortality, especially with respect to differences in magnitude by age, sex, region and time trend.

Information on mortality has recently been added to an SHI claims dataset with complete records of all insured persons in Germany. As almost $90 \%$ of the population is covered by SHI, this data source has enormous potential for public health research, including detailed analyses of mortality patterns. The present study for the first time used outpatient and inpatient SHI claims data drawn from this dataset to analyse observed mortality rates for adults in Germany with and without diagnosed diabetes. Up to now, diabetes-related MRRs from the age of 30 years in 5-year age bands have not been available for the German population. Against this background our main aim was to provide for the first time estimates of MRRs related to diabetes within strata of narrow age bands and sex for Germany, and thus add important knowledge in diabetesrelated excess mortality. Deeply stratified mortality rates based on valid data are important for the surveillance of diabetes in Germany, as they allow a comparison over time and with other countries.

\section{METHODS}

\section{Source of data}

We used the SHI claims research dataset hosted by the German Institute for Medical Documentation and Information (DIMDI). ${ }^{11}$ According to the Data Transparency Regulation Act (DaTraV) 2012, this dataset has been made accessible to authorised health researchers. Originally, these data were collected within the scope of the German morbidity-based risk-adjustment scheme. ${ }^{12}$ The dataset includes medical data from approximately 70 million people covered by SHI, which are about $90 \%$ of the German population. The DaTraV data contain complete data on outpatient and inpatient diagnoses as well as prescribed drugs and the vital status. ${ }^{11}$ Therefore, the data can be analysed across all sectors of care and providers within the SHI system. For reasons of data protection, there is no direct access to these stored individual data. Analyses are limited to aggregate data, which can be requested from the DIMDI data processing centre. A research question needs to be submitted together with an analytical scheme or a syntax query for data analysis. The request has to be approved by the data processing centre and the aggregated results are checked and transmitted to the applicant.

We developed an SQL script for the analysis of mortality rates among persons with and without diabetes based on DaTraV datasets 2013 and 2014. As described in detail below, the SQL script had to take into account several specifics of the data, including assessment of vital status and the case definition for diabetes. ${ }^{13}$

\section{Study population}

Information from more than 70 million SHI persons was available for the year 2013 (figure 1). In addition to the individual SHI identification number, the year of birth and sex were checked for unique assignment to the insured person. Persons with an insured period of less than 360 days, persons who cover at least partly their own health expenditure and persons with main residence

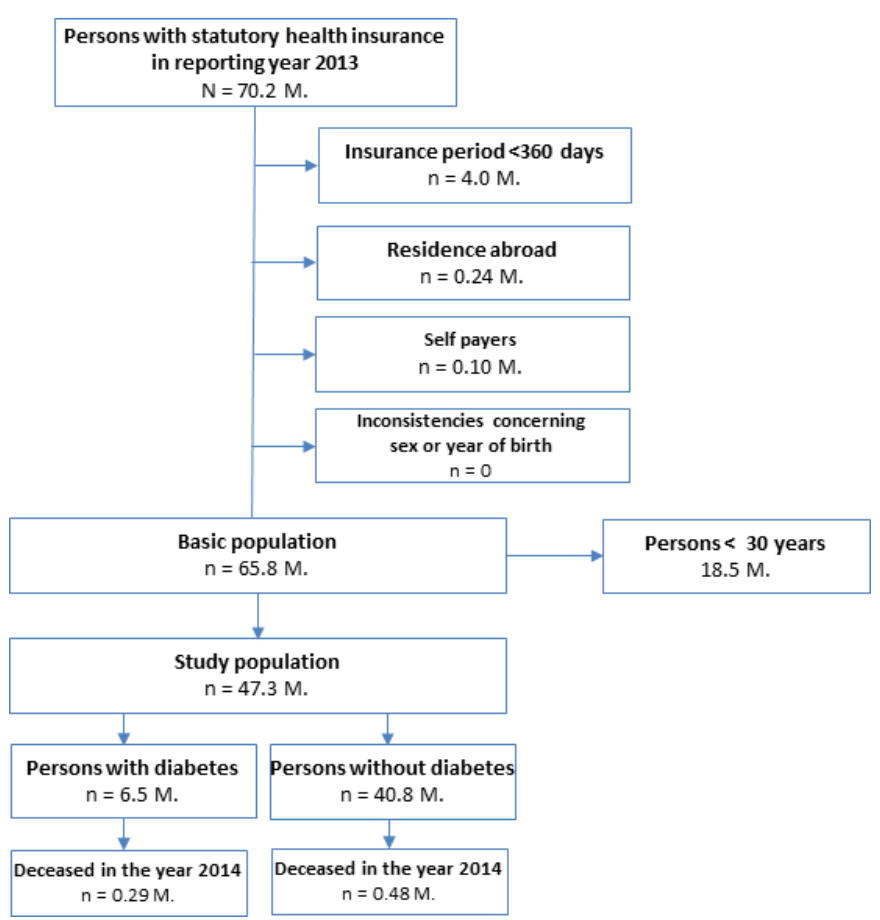

Figure 1 Flow chart of selection of study population with excluding criteria and sample sizes. 
abroad were excluded from the analysis, because this may have precluded documentation of diabetes within the year 2013.

After these exclusions but mainly due to an insurance period of less than 360 days, about 65.8 million persons were considered eligible for analysis. In addition, persons aged younger than 30 years were excluded for data protection reasons due to the small number of deaths among persons with diabetes in these age groups. The final study population hence comprised a total of 47.3 million persons (figure 1). Of these, 6.5 million persons with diabetes fulfilled the case definition for diabetes and 40.8 million persons were defined as having no diabetes. As the flow chart reveals, 0.29 million persons in the population with diabetes and 0.48 million persons in the population without diabetes died in 2014 (figure 1).

\section{Patient and public involvement}

No patient was involved.

\section{Definition of diabetes}

We used the International Classification of Diseases (ICD10) codes E10-E14 to define diabetes:

- E10-type 1 diabetes mellitus.

- E11-type 2 diabetes mellitus.

- E12-malnutrition-related diabetes mellitus.

- E13-other specified diabetes mellitus, for example diabetes related to pancreatic insufficiency.

- E14-unspecified diabetes mellitus.

In the outpatient setting, documentation of an additional ICD-tag ' $G$ ' is required to indicate a confirmed diagnosis of diabetes. In the present analysis, this additional requirement was applied to all data originating in the outpatient setting, in order to increase the validity of the case definition for diabetes. Furthermore, an outpatient diagnosis of diabetes had to be documented in at least two-quarters of the year for validation reasons. This definition is related to the $\mathrm{m} 2 \mathrm{Q}$ criterion, which was originally used for reimbursement and is also recommended for epidemiological studies. ${ }^{14}$ In the case of inpatientdocumented diagnosis, one primary or secondary diagnosis of diabetes in the year 2013 was sufficient to identify a diabetes case.

In order to examine the impact of potential misclassification on the results, we conducted sensitivity analyses applying modified case definitions for diagnosed diabetes based on less stringent criteria: first, documentation of at least one confirmed outpatient diagnosis or one inpatient diagnosis in 2013 ('m1Q criterion'), and second, documentation of only one confirmed outpatient diagnosis in 2013 without any documented inpatient diagnosis.

\section{Assessment of mortality}

We calculated the mortality rates based on the vital status in 2014, since in the event of death no diagnoses for the year of death are available in the dataset. ${ }^{11}$ The reason for this approach is that the SHI claims dataset was originally created only for morbidity-adjusted reimbursement of SHI companies and diagnoses in the year of death were not transmitted. Therefore, we used the difference of the year 2014 and the year of birth to calculate the age groups.

In order to examine whether assessment of vital status in the SHI claims dataset produced plausible results, we compared the observed overall mortality rates as total counts per 100000 persons across age groups and stratified by sex with the corresponding mortality rates from the official cause of death statistics in Germany for the year $2014 .{ }^{15}$ As illustrated in figure 2 mortality rates per 100000 persons based on data from both sources showed high consistency in both sexes and in nearly all age groups, with only minor deviations among middle-aged men and women 85 years of age and older.

\section{Statistical analysis}

We estimated age-specific and sex-specific MRRs and 95\% CIs using Poisson regression. We applied the GENMOD procedure implemented in the statistical software SAS (V.9.4 for Windows). ${ }^{16}$ Due to the aggregated count data of our study population, we applied a count model for MRR estimations. We preferred a Poisson model to a log-binomial model or negative binomial model, as the Poisson distribution provides a good approximation to the underlying binomial distribution due to increasing sample size and better convergence properties. ${ }^{16}$ One central assumption of the model is equality of mean and variance, which is often not fulfilled for count data. In our analyses, we had to handle a large sample size, which tends to result in a lower variance with respect to the mean value, what is called underdispersion and could lead to biased, smaller SEs. Therefore, we used the residual deviance as scale parameter.

We estimated MRRs separately for both sexes and over 5 -year age groups for adults in the age range $30-95$ years and older. We also calculated age-adjusted MRRs stratified by sex based on the 5-year age groups.

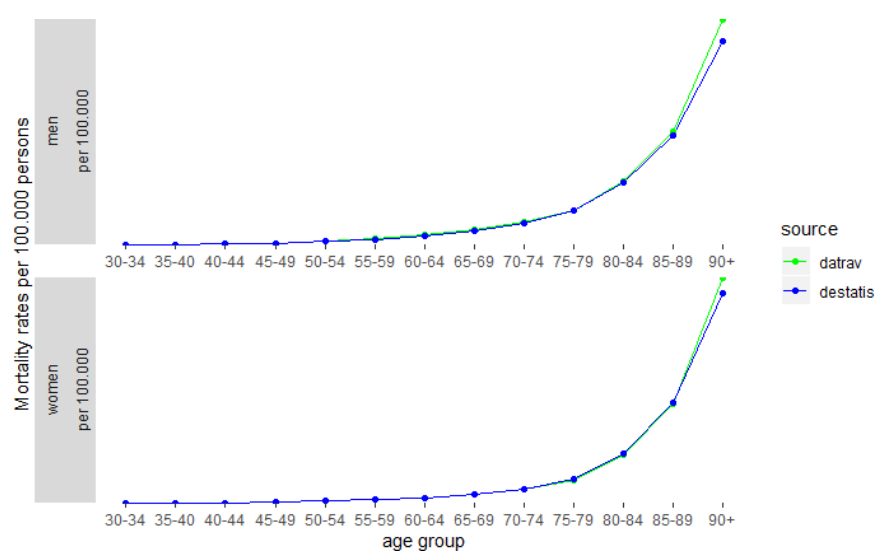

Figure 2 Age-specific mortality rates per 100000 persons stratified by sex for the year 2014 as obtained from official cause of death statistics (Destatis) and claims data (Datrav). The blue line indicates results from official statistics; the green line indicates results from the DaTraV dataset. DaTraV, Data Transparency Regulation Act. 
In order to assess the impact of modified case definitions on the study results, we conducted two sensitivity analyses calculating the age-adjusted MRRs for men and women as described above.

\section{RESULTS}

\section{Description of the study population}

Compared with men, women were over-represented in the population without diabetes, whereas proportions of men and women were similar in the population with diabetes (table 1). Accordingly, the diabetes prevalence among women $(12.8 \%)$ was lower than in men $(14.9 \%)$. As expected, the population with diabetes had a higher mean age compared with the population without diabetes. On average, women were older than men among persons with and without diabetes. In terms of absolute numbers, more women than men died in 2014 in the population with and without diabetes. However, age-specific and age-standardised mortality rates per 1000 persons were

Table 1 Descriptive characteristics of the study population by diabetes status and sex (DaTraV, age $\geq 30$ years)

\begin{tabular}{|c|c|c|c|c|}
\hline & \multicolumn{2}{|c|}{ No diabetes } & \multicolumn{2}{|l|}{ Diabetes } \\
\hline & Women & Men & Women & Men \\
\hline $\begin{array}{l}\text { Population size } \\
\text { in million (2013) }\end{array}$ & 22.5 & 18.3 & 3.3 & 3.2 \\
\hline $\begin{array}{l}\text { Proportion (\%) } \\
2013\end{array}$ & 55.1 & 44.9 & 50.8 & 49.2 \\
\hline $\begin{array}{l}\text { Mean age in } \\
\text { years } 2013\end{array}$ & 55.9 & 53.6 & 71.5 & 67.9 \\
\hline $\begin{array}{l}\text { Number of } \\
\text { deaths } 2014\end{array}$ & 254408 & 220305 & 148491 & 140024 \\
\hline $\begin{array}{l}\text { Mortality rate per } \\
1000 \text { persons }^{*}\end{array}$ & 12.00 & 12.74 & 19.96 & 21.91 \\
\hline \multicolumn{5}{|c|}{ Mortality rate per 1000 persons across age groups* } \\
\hline 30-34 years & 0.30 & 0.62 & 2.03 & 4.26 \\
\hline 35-39 years & 0.45 & 0.87 & 1.86 & 4.38 \\
\hline 40-44 years & 0.77 & 1.40 & 3.75 & 5.31 \\
\hline $45-49$ years & 1.29 & 2.34 & 4.62 & 7.64 \\
\hline $50-54$ years & 2.23 & 4.13 & 6.95 & 10.18 \\
\hline $55-59$ years & 3.41 & 6.73 & 9.19 & 14.55 \\
\hline $60-64$ years & 5.21 & 10.71 & 11.18 & 19.84 \\
\hline $65-69$ years & 7.80 & 15.44 & 15.42 & 25.84 \\
\hline $70-74$ years & 11.63 & 22.35 & 22.56 & 38.24 \\
\hline $75-79$ years & 19.15 & 33.64 & 35.17 & 55.34 \\
\hline 80-84 years & 40.02 & 62.14 & 62.97 & 89.99 \\
\hline 85-89 years & 83.06 & 111.42 & 113.91 & 144.18 \\
\hline 90-94 years & 157.24 & 191.04 & 194.06 & 229.21 \\
\hline $\begin{array}{l}95 \text { years and } \\
\text { older }\end{array}$ & 270.33 & 303.74 & 304.13 & 336.90 \\
\hline
\end{tabular}

*Age standardised to the German population 2013 using all displayed age groups.

DaTraV, Data Transparency Regulation Act. consistently higher among men than women in both populations. In both sexes, mortality rates per 1000 persons were markedly higher among individuals with diabetes than without diabetes (table 1).

\section{Main analysis}

MRR estimates, in association with diagnosed diabetes as obtained from Poisson regression, are depicted in figure 3. For both sexes, the age-specific MRR estimates decreased with increasing chronological age from 6.76 among women and 6.87 among men in the youngest age group to 3.12 among women and 2.46 among men aged 50-54 years to 1.13 among women and 1.11 among men aged 95 years and older. Except for persons younger than 40 years of age, MRR estimates in association with diabetes were higher among women than men. In particular, among persons 50-79 years, the MRR was between 1.26 and 1.12 significantly times higher among women than men.

Overall adjusted MRR estimates were comparable for women and men (1.52 vs 1.56). Constraining our analysis to persons below 90 years of age reversed the overall ageadjusted MRRs regarding sex with still comparable estimates of 1.66 for women and 1.61 for men.

\section{Sensitivity analyses}

An excess risk of death in association with diabetes among men and women was confirmed in two sensitivity analyses applying less stringent case definitions for diabetes (table 2).

Compared with the main analysis, where the case definition for diabetes required documentation of a confirmed diabetes diagnosis in at least two-quarters of the year 2013 for outpatient data or one inpatient diagnosis of diabetes in 2013, the first case definition in table 2 additionally includes persons with only one confirmed outpatient diagnosis of diabetes in 2013. This means that about 0.5 million persons were added to the population with

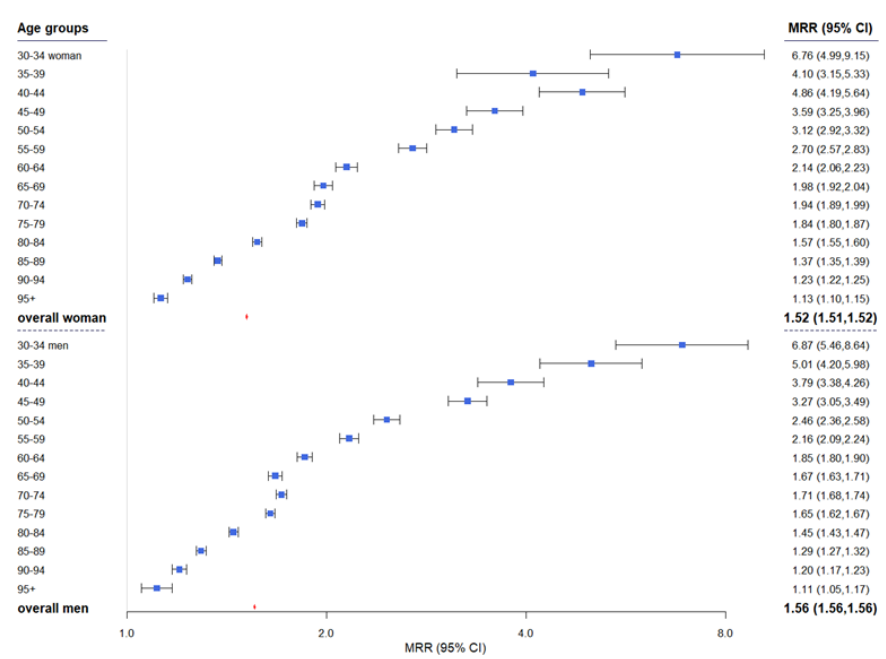

Figure 3 MRRs for persons with diabetes compared with persons without diabetes by sex and age groups. Overall estimates are adjusted using all displayed age groups. MRR, mortality rate ratio. 
diabetes and at the same time removed from the population without diabetes compared with the numbers used for the main analysis as shown in figure 1. Results of this sensitivity analysis were similar to those of the main analysis, with only slightly lower overall MRR estimates of 1.51 among women and 1.55 among men. In contrast, markedly attenuated overall MRR estimates were obtained in the second sensitivity analysis, where the case definition for diabetes was based on the documentation of only one confirmed outpatient diagnosis. Still, the age-adjusted MRRs resulting from this case definition showed a significantly nearly $20 \%$ higher risk of death in men and women with diagnosed diabetes compared with those without diagnosed diabetes (table 2).

\section{DISCUSSION}

\section{Main findings}

To the best of our knowledge, we present for the first time deeply age-stratified MRR estimates in association with diagnosed diabetes among men and women 30 years of age and older in Germany based on SHI claims data covering about $90 \%$ of the population. Overall, men and women with diabetes had an about $50 \%$ higher age-adjusted risk of death compared with adults without diabetes. Across strata of increasing age, the diabetesrelated MRRs considerably decreased with slightly higher estimates among women than men in the population aged $40-80$ years. Results persisted in sensitivity analyses applying modified case definitions for diabetes, with the exception of markedly reduced although still significantly higher diabetes-related risk of death based on the least stringent case definition for diabetes requiring only one outpatient diagnosis for diabetes throughout the year 2013.

Our findings regarding age-related decreases in diabetes-related MRRs partly agree with results from two previous nationwide studies in Germany. ${ }^{1017}$ A populationbased cohort study based on 12-year-mortality follow-up of adults participating in the German National Health Interview and Examination Survey 1998 reported decreasing age-specific diabetes-related MRRs in both sexes as well as overall age-adjusted MRR estimates of similar magnitude as in the present study. ${ }^{17}$ In this previous analysis no sex differences in MRRs from all causes in association with diagnosed type 2 diabetes were observed, although significantly detection of a sex differential may have been precluded by a limited number of deaths among adults with diabetes. Tönnies et al calculated type 2 diabetesrelated MRR applying an illness-death model, with estimates on diabetes prevalence and incidence derived from SHI claims data and mortality rates of the general population from official death statistics. These authors reported age-related decreases in MRRs, but considerably higher overall age-adjusted MRR estimates, with higher estimates among women than men (3.0 vs 2.3) ${ }^{10}$ For comparison with this previous study which focused on the population 65-90 years of age in Germany, we limited our analyses to the population aged 65-90 years and found no differences in MRRs between women and men (1.47 vs 1.48). The study by Jacobs $e t a l^{18}$ calculated, on the basis of the DaTraV dataset as well, the excessive deaths for women and men over 40 years of age in Germany. As no such data were available for Germany at that time, Jacobs $e t$ al took the mortality rates from the Danish National Diabetes Register. The study found absolute excess deaths related to diabetes of 81703 for women and 92924 for men. In contrast, using the same methods but the estimated MRRs for Germany in our study, we found considerably fewer absolute excess deaths of 49136 for women and 53872 for men.

Consistent with our results, nationwide studies in several other countries based on diabetes registers or diabetes surveillance systems have reported a higher diabetesrelated risk of all-cause mortality compared with general population or population-based controls but differ in magnitude. ${ }^{4-68} 19$

The Swedish National Diabetes Register and the Australian diabetes surveillance showed that the excess risk of death in association with diagnosed type 2 diabetes declined with increasing chronological age. ${ }^{68}$ Although the present study could not differentiate by type of diabetes, these results are in line with our findings, since type 2 diabetes accounts for the vast majority of diabetes cases among older adults. The age-related decline in diabetes-related excess risk of all-cause mortality might be due to the different onsets of diabetes on the life span and the associated disease duration. It may reflect increases in competing risk of death in older age groups

Table 2 Sensitivity analyses applying modified diabetes case definitions: number of persons by diabetes status and ageadjusted mortality rate ratios stratified by sex (DaTraV, age $\geq 30$ years)

\begin{tabular}{|c|c|c|c|c|}
\hline & \multicolumn{2}{|l|}{ Women } & \multicolumn{2}{|l|}{ Men } \\
\hline & $\begin{array}{l}\mathrm{N} \text { in million } \\
\text { (no diabetes/ diabetes) }\end{array}$ & $\begin{array}{l}\text { Mortality rate ratios } \\
(95 \% \mathrm{Cl})\end{array}$ & $\begin{array}{l}\mathrm{N} \text { in million } \\
\text { (no diabetes/ diabetes) }\end{array}$ & $\begin{array}{l}\text { Mortality rate ratios } \\
(95 \% \mathrm{Cl})\end{array}$ \\
\hline Sensitivity analysis $2 \dagger$ & $22.3 / 0.25$ & 1.19 (1.18 to 1.20$)$ & $18.1 / 0.21$ & $1.20(1.19$ to 1.21$)$ \\
\hline
\end{tabular}

${ }^{*}$ Documentation of at least one outpatient (confirmed) or inpatient diagnosis of diabetes in 2013.

†Documentation of only one outpatient (confirmed) diagnosis of diabetes in 2013. Deviations from figures in figure 1 are due to rounding. DaTraV, Data Transparency Regulation Act. 
as well as survival disadvantage in association with increased diabetes duration. In addition, the number of severe comorbidities in people with and without diabetes converges with increasing age.

With regard to sex differences in diabetes-related relative risk or excess risk of all-cause mortality, previous studies from other countries showed conflicting findings. ${ }^{458}$ Our age-specific estimates of diabetes-related MRRs showed higher risk estimates among women than among men for persons aged 50-79 years. This higher risk among women declined with increasing age and diminished in the oldest age groups. This consistent pattern is comparable with a study from Australia for 2004-2010 which showed higher standardised mortality ratios (SMRs) in women than in men especially for persons aged 50-79 years and very similar SMRs for women and men aged 80 years or older (1.03 and 0.98). ${ }^{8}$ A recently conducted systematic review and meta-analysis including 49 studies with 86 prospective cohorts showed a combined MRR of 1.93 for women and 1.74 for men with a pooled women-to-men ratio of relative risks (RRR) of 1.13. ${ }^{7}$ However estimates across studies ranged from 1.24 to 3.67 in women and from 1.32 to 3.13 in men, pooled women-to-men RRR varied from 0.64 to $1.74 .^{7}$ Overall, differences in study results regarding a sex differential in excess risk of diabetes-related all-cause mortality might, at least in part, be explained by differences in the age range, underestimation of older people, time of follow-up and applied methods for risk estimation.

Prospective population-based studies are needed to obtain a deeper insight into the role of sex difference in diabetes-related mortality risks by taking relevant risk factors such as lifestyle behaviour, adherence to prescribed therapy and comorbidities into account.

\section{Practical implications}

Our findings confirm that diagnosed diabetes in Germany is still associated with a significantly elevated, several times higher risk of death among men and women, in particular in younger and middle-aged persons. This emphasises the need for effective primary and secondary prevention. Further improvements in the early detection of diabetes, particularly in younger ages, alongside with evidence-based treatments, could contribute to a reduction in excess mortality.

Our results open the perspective to close an important gap in diabetes surveillance in Germany, as the SHI claims dataset appears to be suitable for close monitoring of diabetes-related excess risk of death, which is a key indicator in the national diabetes surveillance system. In addition, the dataset will permit calculation of closely related indicators, including the absolute number of deaths in association with diabetes, and composite indicators of disease burden, including healthy life years and the number of years lost in association with diabetes. ${ }^{20}$ Thus, including SHI claims dataset will harness the potential for improved health information systems as a basis for the surveillance of diabetes and other non-communicable diseases (NCDs).

\section{Strengths and limitations}

The main strength of our analysis is the completeness of the dataset, since about $90 \%$ of the German population is covered by SHI. Mortality rates derived from the SHI claims dataset showed good agreement with data from official death statistics, which underlines the potential for generalisation of our results. Our findings from sensitivity analyses support the validity of the data. We consistently showed an excess risk of all-cause mortality in association with diagnosed diabetes based on varying case definitions for diabetes.

Taken together, our results demonstrate that the DaTraV dataset could essentially contribute to close current gaps in diabetes surveillance with an overall good documentation quality of diabetes and the advantage to consider inpatient as well as outpatient data for case definition.

A great disadvantage of routine datasets based solely on documented diagnoses is that no information about undiagnosed morbidity can be drawn. National surveys with an additional HbA1c measurement in blood samples of participants show a relevant proportion of undiagnosed diabetes. Although this proportion has decreased over time, it is still relatively high ${ }^{21}$ and at the same time is related to a slightly higher excess mortality than diagnosed diabetes. ${ }^{1722}$ For this reason the current routine data analysis is likely to underestimate the excess mortality in diabetes. In general, another limitation of routine data is that these data cannot identify the ethnicity of individuals. An ethnic risk profile is being discussed for diabetes in particular. $^{23}$

There are a number of limitations which arise from the specific construction of the DaTraV dataset, which originally served economic but not research purposes. We had to determine cases of diabetes in the data in 2013 only, in order to identify persons who died in 2014 among persons with and without diabetes. This implies that those who died with newly documented diabetes in 2014 are not detectable in the data as diabetes cases and hence will be counted as persons without diabetes. We cannot exclude that this also contributed to an underestimation of diabetes-related excess mortality. Since diabetes is a chronic disease, and long-term complications account for the majority of diabetes-related deaths, we assume that this had only little impact on our results. The planned revision of the Data Transparency Regulations in Germany could help to overcome current shortcomings.

Furthermore, the currently missing stratification of the dataset according to geographical region and social status or social deprivation should be possible in the future, hence we will be able to analyse and compare mortality trends within Germany as well as at a national level with other countries.

The present study included adults 30 years of age and older, and type 2 diabetes is likely to account for most cases of documented diabetes. Nevertheless, it will be important to overcome current limitations to differentiate between major types of diabetes in claims data. The main problem is the frequent coding of an unspecific diabetes 
(ICD-10: E14) or even diagnoses that are mutually exclusive (E10 and E11) in the data. ${ }^{24} \mathrm{~A}$ recent analysis of the dataset used here has demonstrated that including information on medication may improve assignment of unspecific diabetes codes to type 2 diabetes. ${ }^{25}$ Among children and adolescents, type 1 diabetes is the predominant type of diabetes. As insulin treatment is required here, documented insulin use is an essential part of the case definition for type 1 diabetes, and also helps to clarify diabetes definition. ${ }^{26}$

\section{CONCLUSIONS}

Diabetes-related risk of death is a key indicator for monitoring diabetes epidemiology and quality of diabetes care. Establishing sustainable time trends for this indicator as part of the national diabetes surveillance system in Germany is of great need, but was so far precluded by the lack of a valid and timely accessible dataset. Results of the present study demonstrate that analysis of SHI claims data may provide a solution in closing this information gap. Further research is needed to analyse and to improve the quality of the data, in particular with regard to case definitions. In this case, the SHI claims data could also serve to calculate and monitor the absolute number of diabetes-related deaths as well as composite indicators of disease burden, such as diabetes-related healthy life years and years of life lost. Stratification of SHI claims data according to geographical region and social status or social deprivation will be possible in the future, hence we will be able to analyse and compare diabetesrelated mortality trends not only within Germany but also with international developments. ${ }^{3}$ This will strengthen surveillance activities for the prevention and control of diabetes and other major NCDs at a national level and also enhance international collaboration in diabetes and NCD surveillance and burden of disease estimates.

Contributors CS, JB and CH designed the study. CS, LR and JB performed the analysis. CS, CS-N and JB wrote the initial version of the manuscript. LR, RP and TZ revised the manuscript. All authors read and approved the final manuscript. All authors had full access to all of the data (including statistical reports and tables) in the study and can take responsibility for the integrity of the data and the accuracy of the data analysis. CS is the lead author and guarantor.

Funding The project to establish national diabetes surveillance at the Robert Koch Institute is funded by the German Federal Ministry of Health (funding code: GE 2015 0323 ). The project to promote and further develop the use of external sources of data to secure (establish and expand) national level diabetes surveillance is funded by the Federal Ministry of Health (funding code: GE 201903 05).

Competing interests All authors have completed the Unified Competing Interest form at www.icmje.org/coi_disclosure.pdf (available on request from the corresponding author) and declare no support from any organisation for the submitted work, no financial relationships with any organisations that might have an interest in the submitted work in the previous 3 years, no other relationships or activities that could appear to have influenced the submitted work.

Patient consent for publication Not required.

Provenance and peer review Not commissioned; externally peer reviewed.

Data availability statement No data are available. The SQL code for requesting aggregated data and statistical codes available from the corresponding author at schmidtchri@rki.de.
Open access This is an open access article distributed in accordance with the Creative Commons Attribution Non Commercial (CC BY-NC 4.0) license, which permits others to distribute, remix, adapt, build upon this work non-commercially, and license their derivative works on different terms, provided the original work is properly cited, appropriate credit is given, any changes made indicated, and the use is non-commercial. See: http://creativecommons.org/licenses/by-nc/4.0/.

\section{ORCID iDs}

Christian Schmidt http://orcid.org/0000-0002-8419-8856

Christin Heidemann http://orcid.org/0000-0002-9413-2148

Rebecca Paprott http://orcid.org/0000-0003-2919-450X

Jens Baumert http://orcid.org/0000-0003-1399-6874

\section{REFERENCES}

1 NCD Risk Factor Collaboration (NCD-RisC). Worldwide trends in diabetes since 1980: a pooled analysis of 751 population-based studies with 4.4 million participants. Lancet 2016;387:1513-30.

2 Williams J, Loeffler M, Metrics H, Institute for Health Metrics and Evaluation. Global trends in type 2 diabetes, 2007-2017. JAMA 2019;322:1542-42.

3 Harding JL, Pavkov ME, Magliano DJ, et al. Global trends in diabetes complications: a review of current evidence. Diabetologia 2019;62:3-16.

4 Green A, Sorts $\varnothing$ C, Jensen PB, et al. Incidence, morbidity, mortality, and prevalence of diabetes in Denmark, 2000-2011: results from the diabetes impact study 2013. Clin Epidemiol 2015;7:421-30.

5 Read SH, Kerssens JJ, McAllister DA, et al. Trends in type 2 diabetes incidence and mortality in Scotland between 2004 and 2013. Diabetologia 2016;59:2106-13.

6 Tancredi M, Rosengren A, Svensson A-M, et al. Excess mortality among persons with type 2 diabetes. $N$ Engl J Med 2015;373:1720-32

7 Wang Y, O'Neil A, Jiao Y, et al. Sex differences in the association between diabetes and risk of cardiovascular disease, cancer, and all-cause and cause-specific mortality: a systematic review and meta-analysis of 5,162,654 participants. BMC Med 2019;17:136.

8 Harding JL, Shaw JE, Peeters A. Mortality trends among people with type 1 and type 2 diabetes in Australia: 1997-2010. Diabetes Care 2014:DC_140096.

9 Heidemann C, Scheidt-Nave C. Prevalence, incidence and mortality of diabetes mellitus in adults in Germany - a review in the framework of the diabetes Surveillanc: Robert Koch-Institut, Epidemiologie und Gesundheitsberichterstattung 2017.

10 Tönnies T, Hoyer A, Brinks R. Excess mortality for people diagnosed with type 2 diabetes in 2012 - Estimates based on claims data from 70 million Germans. Nutr Metab Cardiovascul Dis 2018;28:887-91.

11 German Institute of Medical Documentation and Information (DIMDI). Health care data. Available: https://www.dimdi.de/dynamic/en/ further-services/health-care-data/

12 Busse R, Blümel M, Knieps F, et al. Statutory health insurance in Germany: a health system shaped by 135 years of solidarity, selfgovernance, and competition. Lancet 2017;390:882-97.

13 Schmidt C, Heidemann C, Rommel A. Secondary data in diabetes surveillance - co-operation projects and definition of references on the documented prevalence of diabetes. J Health Monitoring 2019;4:50-63.

14 Schubert I, Ihle P, Köster I. [Internal confirmation of diagnoses in routine statutory health insurance data: concept with examples and case definitions]. Gesundheitswesen 2010;72:316-22.

15 Statistisches Bundesamt. Fachserie 12 Reihe 4. Gesundheit. Todesursachen in Deutschland, 2014. Available: https://www. destatis.de/GPStatistik/receive/DEHeft heft 00035170

16 Fang J. Using SAS® procedures FREQ, GENMOD, logistic, and PHREG to estimate adjusted relative risks - a case study. SAS globa forum 2011.

17 Röckl S, Brinks R, Baumert J, et al. All-Cause mortality in adults with and without type 2 diabetes: findings from the National health monitoring in Germany. BMJ Open Diabetes Res Care 2017;5:e000 451-e51.

18 Jacobs E, Hoyer A, Brinks R, et al. Burden of mortality attributable to diagnosed diabetes: a nationwide analysis based on claims data from 65 million people in Germany. Diabetes Care 2017;40:1703-9.

19 Gregg EW, Cheng YJ, Srinivasan M, et al. Trends in cause-specific mortality among adults with and without diagnosed diabetes in the USA: an epidemiological analysis of linked national survey and vital statistics data. Lancet 2018;391:2430-40. 
20 Baumert J, von der Lippe E, Paprott R, et al. Life expectancy and healthy life years associated with diabetes in Germany. Eur J Public Health 2020;30.

21 Heidemann C, Du Y, Paprott R, et al. Temporal changes in the prevalence of diagnosed diabetes, undiagnosed diabetes and prediabetes: findings from the German health interview and examination surveys in 1997-1999 and 2008-2011. Diabet Med 2016;33:1406-14.

22 Paprott R, Schaffrath Rosario A, Busch MA, et al. Association between hemoglobin A1c and all-cause mortality: results of the mortality follow-up of the German National health interview and examination survey 1998. Diabetes Care 2015;38:249-56.
23 Xu G, Liu B, Sun Y, et al. Prevalence of diagnosed type 1 and type 2 diabetes among US adults in 2016 and 2017: population based study. BMJ 2018;362:k1497.

24 Tamayo T, Brinks R, Hoyer A, et al. The prevalence and incidence of diabetes in Germany. Dtsch Arztebl Int 2016;113:177-82.

$25 \mathrm{Ct} \mathrm{T}$, Schwalm A, Mostardt S. Can routine data be used to determine the target population of patients with type 2 diabetes in early benefit assessments in Germany? Gesundheitswesen (Bundesverband der Arzte des Offentlichen Gesundheitsdienstes).

26 Bohn B, Schwandt A, Ihle P, et al. Hospital admission in children and adolescents with or without type 1 diabetes from Germany: an analysis of statutory health insurance data on 12 million subjects. Pediatr Diabetes 2018;19:721-6. 